\title{
Increasing Phenotypic and Genetic variations in Hyperactivity/Inattention Problems from Age 3 to 13 Years: A Cross-Sectional Twin Study
}

\author{
Yoon-Mi Hur \\ Department of Education, Mokpo National University, South Korea
}

\begin{abstract}
A twin design was used to examine the developmental nature of genetic, environmental, and phenotypic variations in hyperactivity and inattention problems (HIP). Mothers of 662 complete pairs of twins (273 monozygotic [MZ] pairs and 389 dizygotic [DZ] pairs) aged from 3 to 13 years (mean [SD] age $=8.3$ [2.9] years) responded to the items of the HIP scale of the Strengths and Difficulties questionnaire via a telephone interview. Maximum likelihood $\mathrm{MZ}$ and $\mathrm{DZ}$ twin correlations in the total sample were 0.47 (95\% Cl: $0.37-0.55)$ and -0.01 (95\% Cl: $-0.11-0.09)$. A standard univariate model incorporating age as a modifier was applied to the raw data. Results of model-fitting analyses showed that the phenotypic variation of HIP monotonically increased from age 3 to age 12 and that this increase was completely due to an increase in genetic variance, suggesting that it is genes that expand individual difference in ADHD symptoms with age during childhood. Child-specific environmental variance was constant during this age period. In terms of relative influences, total genetic factors increased from 33\% ( $95 \% \mathrm{Cl}: 27-44 \%$ ) at age 3 to $51 \%$ (95\% Cl: $28-71 \%$ ) at age 13 and this increase was accompanied by a decrease in relative influences of child-specific environmental factors from $67 \%$ (95\% Cl: $56-73 \%)$ at age 3 to $49 \%$ (95\% Cl: $29-72 \%)$ at age 13. These estimates of genetic influences were somewhat lower than those found in most twin studies of ADHD symptoms. However, the increasing trend of genetic influences with age during childhood was consistent with the results of a recent meta-analysis of ADHD symptoms.
\end{abstract}

Keywords: hyperactivity, ADHD, twin, genetics, children, development

Hyperactivity and inattention are core symptoms of Attention Deficit/Hyperactivity Disorder (ADHD), one of the most commonly diagnosed neurobehavioral disorders of childhood (American Psychiatric Association, 2000). Hyperactivity and inattention problems (HIP) are known to be associated with difficulties in adjustment during childhood and adolescence, such as academic failures and problems with peer and family relationships (Taylor, 1994). The worldwide-pooled prevalence of ADHD in children has been estimated to be 5.29\% (95\% CI: 5.01-5.56; Polanczyk et al., 2007). Twin and family studies have suggested that HIP are strongly heritable. However, heritability estimates reported in the literature vary considerably: Depending on age of the sample, raters and measures used in the study, and whether twins are rated by the same or different informants and in the same or different contexts, heritability estimates were found to be up to about $80 \%$ (Chang et al., 2013, Freitag et al., 2010; Hay et al., 2007; Kan et al., 2014; Rietveld et al., 2004). Shared family environmental influences were negligible, and environmental influences on HIP were predominantly of the non-shared type; that is, environmental factors specific to each child (Nikolas \& Burt, 2010). The importance of dominance genetic effects were also suggested (Nikolas \& Burt, 2010). However, several researchers have argued that rater bias may partly represent dominance genetic effects found in childhood HIP (Rietveld et al., 2003; Saudino et al., 2000; Simonoff et al., 1998).

HIP typically begin during childhood, but they can start during adolescence as well, and persist into adulthood (Faraone et al., 2006). There are large individual differences in the developmental pattern of HIP, where the problems

RECEIVED 26 May 2014; ACCEPTED 25 August 2014. First published online 3 October 2014.

ADDRESS FOR CORRESPONDENCE: Yoon-Mi Hur, Department of Education, Mokpo National University, South Korea. E-mail:ymhur@mokpo.ac.kr 
increase from childhood to adolescence in some children, but diminish over time in others (Robbers et al., 2011). These complex developmental trajectories suggest that genetic and environmental sources of HIP may change during childhood and adolescence. Several longitudinal studies of HIP examined whether genetic and environmental influences continue or change across development. For example, Larsson et al. (2004) studied the developmental change of ADHD in a nationwide longitudinal sample of Swedish twins at two points of measurement: 8 to 9 years, and 13 to 14 years. Significant heritabilities in ADHD symptoms were found at both time points, with the estimates being $68 \%$ for girls and $35 \%$ for boys at wave 1 , and $74 \%$ for boys and $61 \%$ for girls at wave 2. Continuity, as well as changes in symptoms over the 5-year period, was also demonstrated. The continuity was mainly due to the same genetic effects operating at both time points, whereas changes were attributable to new genetic and new child-specific environmental factors that emerged during early adolescence.

In another large longitudinal sample, Rietveld et al. (2004) analyzed genetic and environmental influences on Overactivity (OA) and Attention Problems (AP) in the Child Behavior Checklist (CBCL), measured at ages 3, 7, 10 , and 12 years. The scores of OA and AP from age 3 to age 12 years remained stable, which was largely due to additive genetic influences common across the age groups studied. The estimate of heritability at each age group was the same (approximately 75\%). However, the genetic overlap across the age groups was not complete, suggesting that new genetic influences may show up during development. These results were close to the findings from a large, longitudinal study of UK twins (Kuntsi et al., 2005) where the Revised Rutter Parent Scale for Pre-School Children, Strengths and Difficulties Questionnaire (SDQ) used in the present study, and Conners' DSM-IV ADHD Symptom Scores were given to twins at ages 2, 3, 4, 7, and 8 years. Heritability estimates at each age were substantial and phenotypic correlations across the age groups were significant, indicating stability of the symptoms. Longitudinal analyses revealed that shared genetic influences largely explained the phenotypic stability $(59 \%$ to $96 \%)$. Child-specific environmental influences overall were small and contributed more toward change than stability across age groups. Cholesky decomposition analyses confirmed that at each age, new genetic influences showed up. The authors also found that the magnitudes of genetic and environmental influences on ADHD symptoms were similar in boys and girls.

Although most longitudinal twin studies demonstrated emergence of new genetic and new child-specific environmental effects on HIP during childhood and adolescence, very few twin studies thus far have paid attention to the issue of whether phenotypic variation of HIP becomes larger with these increasing genetic and environmental effects, as most twin studies have focused on relative influences of genetic and environmental factors in HIP. Indeed, a gen- eral increase of phenotypic variation of HIP with age was reported in Kunti et al. (2005; see Table 4) and Rietveld et al. (2004; see Table 2).

During childhood and adolescence, children may become more variable on HIP with increasing age as a result of accumulation of experiences, such as transition to school, academic challenges, and development of social relationships with peers, teachers, and others outside the family. Behavioral genetic methods allow us to explain underlying causes of age differences in phenotypic variabilities by partitioning the total variance into genetic and environmental sources. There are two aims in the present study: (1) to investigate whether phenotypic variance in HIP increases from early childhood to early adolescence, and if so, (2) to explore genetic and environmental sources associated with the increases in the phenotypic variance.

\section{Methods}

\section{Sample}

The present sample consisted of 662 complete pairs of twins (273 monozygotic [MZ] pairs and 389 dizygotic [DZ] pairs) drawn from the South Korean Twin Registry (SKTR; Hur et al., 2013). The SKTR is a nationwide volunteer twin registry that includes twins from infants to young adults. In the SKTR, twins are mainly recruited from schools, large maternity hospitals, and twin mothers' clubs on the internet. Higher number of DZ than MZ twins in the present sample reflected a recent sharp increase of DZ twin births in South Korea (Hur \& Song, 2009). Twins used in the present study ranged in age from 3 years to 13 years (mean $=8.3$ years, $S D=2.9$ years; see Table 1 ).

Twins' zygosity was determined from mothers' responses to a zygosity questionnaire that includes questions regarding physical similarities and frequency of confusion of the twins by family members and others (Ooki et al., 1993). To minimize misclassification of zygosity, we excluded twins whose zygosity was ambiguous.

\section{Measure}

To measure the HIP of twins, a Korean version of the SDQ (Ahn et al., 2003; Goodman, 1997) was given to mothers of twins through a telephone interview. SDQ is one of the most commonly used instruments to measure mental health problems in children and adolescents aged from 3 to 17 years, and has been translated into over 70 languages worldwide. It includes 25 items to represent five scales (Emotional Problems, Peer Problems, Hyperactivity/Inattention Problems, Conduct Problems, and Prosocial Behavior). Confirmatory factor analyses based on the item covariance matrix has shown the five-factor solution in line with the five scales of the SDQ and the factor loadings were shown to be generally similar across different age groups (Stone et al., 2010). A review that examined 48 studies $(N=131,223)$ regarding psychometric properties of the SDQ made a conclusion that internal consistency and 
TABLE 1

Sample Size, Mean (SD) for Age, and Maximum Likelihood MZ and DZ Twin Correlations and Mean (SD) for Hyperactivity and Inattention Problems (HIP)

\begin{tabular}{lllll}
\hline & Sex & MZ & DZ & Total \\
\hline$N$ & $M$ & 298 & 435 & 733 \\
& $\mathrm{~F}$ & 248 & 345 & 593 \\
Age in years & & & & \\
Mean (SD) & $\mathrm{M}$ & $8.85(2.83)$ & $7.92(2.88)$ & $8.89(2.89)$ \\
& $\mathrm{F}$ & $9.46(2.97)$ & $8.17(2.85)$ & $8.89(2.90)$ \\
HIP & & & & $3.60(2.63)$ \\
Mean (SD) & $\mathrm{M}$ & $3.75(2.66)$ & $3.49(2.61)$ & $2.32(2.22)$ \\
& $\mathrm{F}$ & $2.49(2.14)$ & $2.21(2.16)$ & \\
$r$ & & $0.47(95 \% \mathrm{Cl}: 0.37-0.55)$ & $-0.01(95 \% \mathrm{Cl}:-0.11-0.09)$ & \\
\hline
\end{tabular}

Note: $M Z=$ monozygotic twins, $D Z=$ dizygotic twins, $M=$ male, $F=$ female.

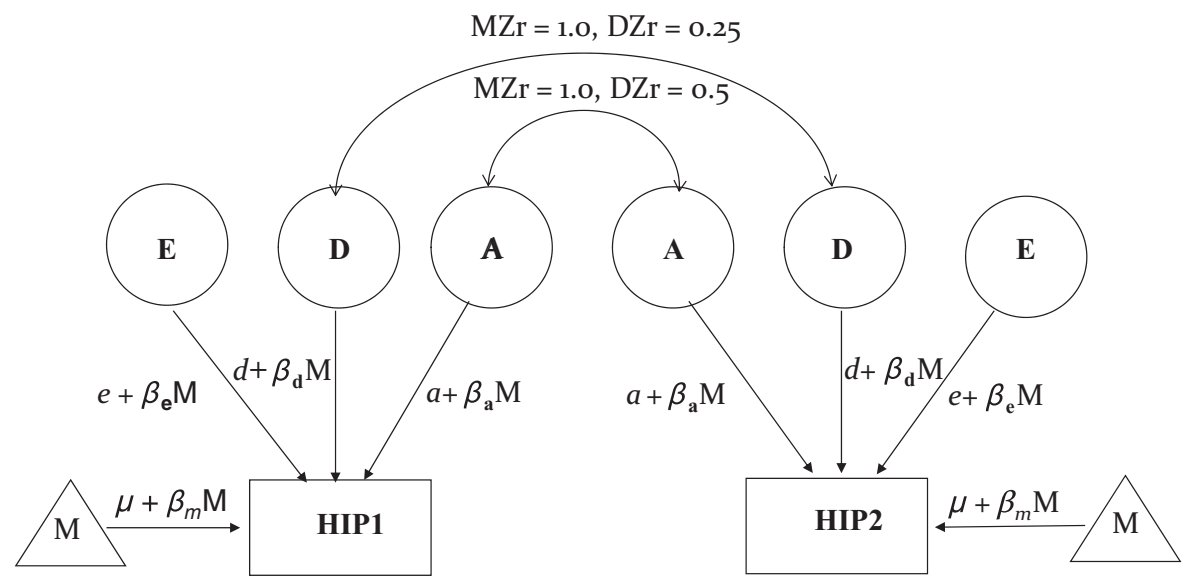

\section{FIGURE 1}

Additive genetic (A), nonadditive genetic (D), and child-specific environment and error (E) effects on the scores of the HIP. Subscripts 1 and 2 refer to the first- and the second-born twin, respectively. Note: HIP = Hyperactivity and Inattention Problems. M = moderator (age), $\boldsymbol{\beta}=$ regression coefficient for $M$.

test-retest reliabilities and validity of the five scales are satisfactory across different age groups and sexes (Stone et al., 2010).

The HIP scale of the SDQ includes five items: 'Restless, overactive, cannot stay still for long'; 'Constantly fidgeting or squirming; easily distracted', 'Concentration wanders; thinks things out before acting'; 'See work through to the end, good attention span'. Mothers of twins were asked to rate each item on a 3 -point Likert scale $(0=$ not true, $1=$ somewhat true, $2=$ certainly true). The internal consistency reliability of the five items was 0.75 in the present sample.

\section{Statistical Analyses}

A standard univariate twin model incorporating age as a moderator (age-moderation model) was applied to the raw data (Figure 1; Purcell, 2002). The age-moderation model includes additive genetic factors (A) (i.e., the sum of the average effect of all alleles that influence a trait), nonadditive genetic factors (D) (i.e., the effect of alleles arising from intralocus and interlocus interactions, and child-specific environmental factors, and (E) (i.e., those environmental factors unique to each member of a twin pair and measurement error). Shared environmental factors were not included in the model because twin correlations (see Table 1) suggested no evidence for shared environmental factors for HIP, which was consistent with literature of ADHD symptoms. Additive genetic factors (A) are set to be correlated at 1.0 and 0.5 for $\mathrm{MZ}$ and $\mathrm{DZ}$ twins, respectively, and nonadditive genetic factors (D), at 1.0 and 0.25 , respectively. Nonshared environmental factors (E) are uncorrelated between two members of a twin pair.

The age-moderation model includes the standard paths, a, $d$, and e, indicating the magnitude of moderation effects related to $\mathrm{A}, \mathrm{D}$, and $\mathrm{E}$, respectively, which are allowed to vary as a function of a moderator, age (M). The phenotypic variance $(\mathrm{Vp})$ in the age-moderation model can be expressed as: $\mathrm{Vp}=\left(\mathrm{a}+\beta_{\alpha} \mathrm{M}\right)^{2}+\left(\mathrm{d}+\beta_{\mathrm{d}} \mathrm{M}\right)^{2}+\left(\mathrm{e}+\beta_{\mathrm{e}} \mathrm{M}\right)^{2}$. In this equation, $\beta_{\alpha}, \beta_{d}$, and $\beta_{\mathrm{e}}$ represent the magnitude of the moderating effects associated with $\mathrm{A}, \mathrm{D}$, and $\mathrm{E}$, respectively. Thus, for example, if $\beta_{\alpha}$ is significantly different from zero, then the influence of additive genetic factors varies as a function of age. The same applies to $\beta_{\mathrm{d}}$ and $\beta_{\mathrm{e}}$. In addition, we allowed $a_{\mu}+\beta_{m} M$ to represent the main effects of age on HIP. 


\begin{tabular}{|c|c|c|c|c|c|c|c|}
\hline \multicolumn{8}{|c|}{$\begin{array}{l}\text { TABLE } 2 \\
\text { Model-Fitting Results }\end{array}$} \\
\hline Model & Description & $-2 \mathrm{LL}$ & AIC & $d f$ & $\Delta-2 \mathrm{LL}$ & $\Delta d f$ & $p$ \\
\hline Full & $A, D, E, a, d, e$ & 3687.8 & 1055.8 & 1316 & & & \\
\hline 1 & $A, E, a, e$ & 3698.8 & 1062.8 & 1316 & 10.9 & 2 & .00 \\
\hline 2 & $A, D, E, d, e$ & 3687.8 & 1053.8 & 1317 & 0 & 1 & 1.00 \\
\hline 3 & $A, D, E, e$ & 3697.0 & 1061.0 & 1318 & 9.1 & 2 & .01 \\
\hline 4 & $A, D, E, d$ & 3692.3 & 1056.3 & 1318 & 4.4 & 2 & .11 \\
\hline 5 & $A, D, E$ & 3697.0 & 1059.0 & 1319 & 9.1 & 3 & .03 \\
\hline
\end{tabular}

The maximum likelihood raw data option in Mx (Neale et al., 2003) was used in our model-fitting analyses. Mx calculates twice the negative log-likelihood (-2LL) of the data. As the difference in -2LL is chi-square distributed, when models were nested to each other, the likelihood ratio test (LRT) was used to evaluate alternative models. When models were not nested to each other, Akaike's information criterion (AIC $=-2 \mathrm{LL}-2 \mathrm{df}$ ) for alternative models were compared. Models having lower AIC are considered more parsimonious, and thus preferred (Akaike, 1987). A saturated model was created for a baseline comparison. In the saturated model, means, variances, and covariances were set to vary across zygosity groups. To determine the bestfitting, most parsimonious model, parameters in the full model were constrained, and the resulting goodness-of-fit of the reduced models were compared with that of the full model.

\section{Results}

\section{Descriptive Statistics and Twin Correlations}

The distribution of HIP was somewhat positively skewed with a skewness of 0.79 and a kurtosis of -0.12 . However, as the skewness and kurtosis were not seriously high, transformation of the score was not necessary. Means and SDs of HIP for MZ and DZ twins are presented in Table 1. Age was not significantly correlated with the score of HIP in the present sample. However, males had higher mean score and variance than females for HIP. $(t=9.7, p<.01 ; F=41.9$, $p<.01)$. Within each sex, twins were not significantly different across zygosity groups in the mean level or variance of HIP, indicating the absence of zygosity effects. No significant mean or variance difference was found between the first- and the second-born twins, suggesting no birth order effects on HIP. Overall, these results fulfilled assumptions for analysis of twin data.

Maximum likelihood $\mathrm{MZ}$ and $\mathrm{DZ}$ twin correlations were 0.47 (95\% CI: 0.37-0.55) and -0.01 (95\% CI: -0.11-0.09), respectively (Table 1). This pattern of twin correlations suggested the importance of genetic influences on HIP. In addition, much lower $\mathrm{DZ}$ than half of MZ twin correlations indicated the presence of nonadditive genetic rather than shared environmental factors in HIP.

\section{Model-Fitting Analyses}

Selection of the best-fitting model. The difference in -2 LL between the saturated and the full model was not significant $\left(\Delta \mathrm{X}^{2}=6.6, \Delta \mathrm{df}=6, p>.25\right)$, indicating that the restrictions made in the full model are acceptable. Table 2 provides goodness-of-fit indices of the full model and its nested models. Non-additive genetic factors (D) and the associated age moderator (d) were first removed simultaneously from the full model (Model 1). This procedure significantly worsened the model-fit. In Model 2, the age moderator on additive genetic factors (a) was eliminated from the full model, which resulted in a non-significant change in -2LL. Next, the age moderator on non-additive genetic factors (d) and child-specific environmental factors (e) was individually removed from Model 2. A significant change in -2LL occurred in the former (Model 3) but not in the latter procedure (Model 4), suggesting that it is necessary to retain the age moderator on non-additive genetic factors (d). From these model comparisons, it was concluded that Model 4 was the best-fitting one, where the age moderator on nonadditive genetic factors (d), in addition to additive and nonadditive genetic factors (A) and (D) and child-specific environmental factors including measurement error (E), remained significant. One should note that A was not removed from the full model because it has been argued that an extreme amount of nonadditive genetic effects with no effects of additive genes is unlikely to explain genetic variance for complex traits (Eaves, 1988).

Parameter estimates in the best-fitting model. Figures 2 and 3, respectively, present graphical descriptions of unstandardized and standardized variance components in the best-fitting model for HIP. It should be emphasized that as the present sample did not have sufficient statistical power to distinguish between additive and nonadditive genetic variance, all genetic variance was absorbed into nonadditive variance component, leading the estimate of additive genetic variance to be zero. For this reason, additive and nonadditive genetic variances were summed to represent 


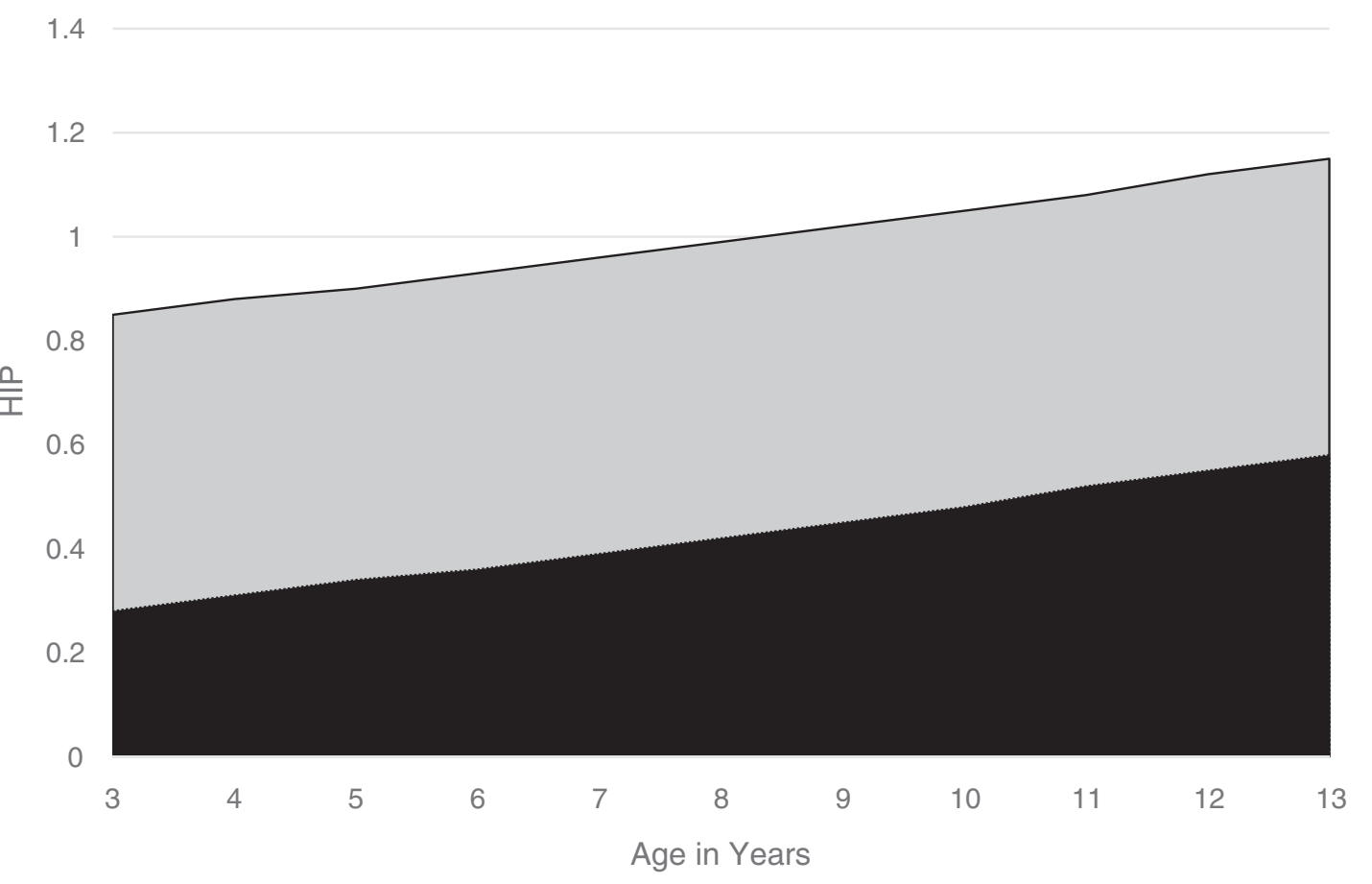

\section{FIGURE 2}

Unstandardized variance of additive and nonadditive genetic factors ( $A+D$; black area) and child-specific environmental factors including measurement error ( $E$; grey area) and total phenotypic variance in Hyperactivity and Inattention Problems from age 3 to 13 years.

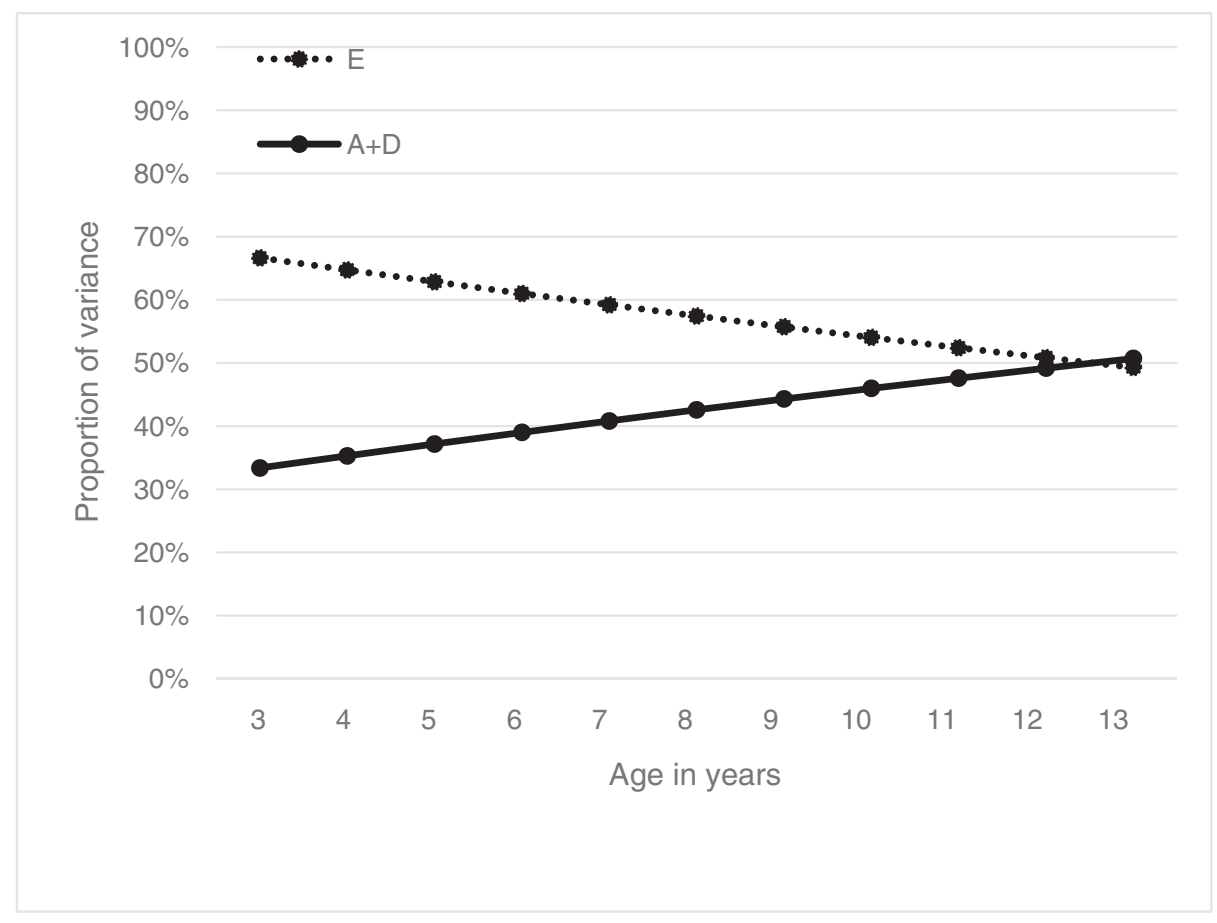

\section{FIGURE 3}

Relative influences $(\%)$ of additive and nonadditive genetic $(A+D)$ and child-specific environmental factors including measurement error (E) in Hyperactivity and Inattention Problems from age 3 to 13 years. 
broad-sense heritability, that is, total genetic variance in Figures 2 and 3. Figure 2 shows that total phenotypic variance for HIP increased from 0.85 at age 3 years to 1.15 at age 13 years. A parallel increase occurred in total genetic variance $(A+D)$ from 0.28 at age 3 years to 0.58 at age 13 years, whereas child-specific environmental variance $(0.57)$ was constant during this period. These results indicated that the increase in the phenotypic variance of HIP was due completely to an increase in total genetic variance.

In terms of relative influences, total genetic factors increased from 33\% (95\% CI: $27-44 \%$ ) at age 3 to $51 \%$ (95\% CI: $28-71 \%)$ at age 13 , and this increase was accompanied by a decrease in relative influences of child-specific environmental factors from $67 \%$ (95\% CI: $56-73 \%$ ) at age 3 to 49\% (95\% CI: 29-72\%) at age 13 (Figure 3).

\section{Discussion}

The present study showed that individual differences in HIP monotonically increased from early childhood to early adolescence and that this increase was driven almost exclusively by an increase in genetic differences. These findings support the results from prior longitudinal twin studies that demonstrated emergence of additional genetic factors across development (Kuntsi et al., 2005; Rietveld et al., 2004). Increasing genetic variation found in the present cross-sectional examination may represent additional genetic risk factors that emerged with age, reported in previous longitudinal analyses of childhood twins. Note, however, that the increasing trend of genetic and phenotypic variance of HIP may not be observed in adulthood. In a review of findings on ADHD symptoms in Dutch twin children, adolescents, and adults, Kan et al. (2013) reported a notable decrease in genetic variance in adulthood.

The estimates of relative influences of genetic factors found in this study are generally at the lower side of those reported in twin studies of ADHD symptoms. Although the internal consistency reliability of the HIP scale in the present sample was acceptable $(\alpha=0.75)$, the number of items in the scale was short, and thus, the scale was limited in measuring HIP, which may have contributed to high child-specific environmental influences found in this study. Replications of the present findings with different instruments, as well as larger samples, are clearly necessary. Note, however, that the increasing pattern of relative influences of genetic factors with age reported in this study is consistent with the results of a recent meta-analysis of ADHD symptoms (Nikolas \& Burt, 2010). Nikolas and Burt reviewed approximately 80 published twin and adoption studies of inattention and hyperactivity and concluded that relative influences of total genetic factors $(A+D)$ increased from $62 \%$ in early childhood ( $0-5$ years) to $74 \%$ in late childhood (6-11 years) for inattention, and from $66 \%$ to $82 \%$ in the corresponding age groups for hyperactivity.
Genetic variance for the increase in HIP from early childhood to early adolescence may in part represent the genetic covariance of HIP with many other psychological and psychiatric traits. It is well established that ADHD symptoms are correlated with many psychiatric and psychological traits. For example, a population-based twin study reported that up to $90 \%$ of the children with ADHD were affected by at least one comorbid disorder (Willcutt et al., 1999). In addition, ADHD symptoms have been shown to be correlated with IQ (Kuntsi et al., 2004), temperament (Kerekes et al., 2013), and mood (Cole et al., 2009) among others. It is well documented that heritability for these traits tend to increase from childhood to adolescence (Plomin et al., 2001). Thus, some of the genetic variance increasing with age found in the present study may include increasing genetic variances for these correlates of ADHD symptoms. Indeed, recent twin studies showed that common genetic factors substantially underlie the relationship of ADHD with ODD/CD (Nadder et al., 2002) and with IQ (Kuntsi et al., 2004). More multivariate twin studies using ADHD symptoms and related psychological and psychiatric traits are necessary to elucidate the nature of the increase in genetic variance in HIP from childhood to early adolescence found in the present sample.

Although the results of GWAS and candidate gene studies of ADHD thus far were not consistent, recent reviews (Faraone \& Mick, 2011; Thapar et al., 2012) concluded that the dopamine transporter genes DAT1 and SLC6A3, the dopamine receptor genes DRD4 and DRD5 and SNAP25 on chromosome 20p12, and the serotonin receptor geneHTR1B may be associated with the susceptibility to ADHD. The reviews further suggested that many genes of small effect size may influence the risk of ADHD. The increase of genetic variance with increasing age found in the present study supports the presence of age-specific genes for ADHD, emphasizing that it is important for molecular genetic researchers to search genes for ADHD using subjects at a similar developmental stage. The present results also suggest that in developing intervention and prevention programs for ADHD for children and adolescents, age should be incorporated for the programs to be more effective.

There are several limitations in the present study. First, only maternal rating was used to measure twins' HIP. Mothers have been reported to exaggerate dissimilarity of DZ twins (sibling-contrast effects), which may have caused overestimation of nonadditive genetic contributions and underestimations of shared environmental contributions to HIP (Saudino et al., 2000). Sibling-contrast effects are often manifested with larger DZ than MZ twin variances and strong negative DZ twin correlations. In the present study, the variance of HIP was not significantly different between $\mathrm{MZ}$ and DZ twins, and DZ twin correlation was -0.01 (Table 1), suggesting that the effects may not be very serious. However, future investigations should employ multiple raters to determine the extent to which sibling contrast 
effects influence parameter estimates. Second, the model used in this study assumed no genotype-environment interactions or correlations for HIP. However, recent evidence suggested that environmental adversity increased genetic risk for externalizing problems in adolescents (Hicks et al., 2009). Thus, future studies will have to incorporate environmental variables in the model to better understand genetic and environmental etiologies of ADHD symptoms during childhood and adolescence. Third, the present study is a cross-sectional rather than longitudinal examination. However, the age was treated as a continuous rather than categorical variable, which can provide the most sensitive test for age differences in genetic, environmental, and phenotypic variation in HIP during childhood and early adolescence. Finally, the size of the present twin sample was not large enough to fit the age-moderation model separately for males and females, which could increase our understanding of the source of sex difference in the variance of HIP. However, several prior twin studies of ADHD symptoms demonstrated none or relatively small sex differences in the magnitudes of genetic and environmental influences during childhood and adolescence (Kuntsi et al., 2005; Rietveld et al., 2004).

\section{Acknowledgments}

This study was supported by the Pioneer Fund, USA, Charles Darwin Research Institute, USA, Ulster Institute for Social Research, UK, and National Research Foundation of Korea (NRF-371-2011-1 B00047). I would like to thank twins and their parents who participated in this study.

\section{References}

Ahn, J.-S., Jun, S.-K., Han, J.-K., Noh, K.-S., \& Goodman, R. (2003). The development of a Korean version of the strengths and difficulties questionnaire. Journal of Korean Neuropsychiatric Association, 42, 141-147.

Akaike, H. (1987). Factor analysis and AIC. Psychometrika, 52, 317-332.

American Psychiatric Association. (2000). Diagnostic and statistical manual of mental disorders (4th ed., DSM-IV-TR). Washington, DC: American Psychiatric Association.

Chang, Z., Lichtenstein, P., Asherson, P. J., \& Larsson, H. (2013). Developmental twin study of attention problems: High heritabilities throughout development. JAMA Psychiatry, 70, 311-318.

Cole, J., Ball, H. A., Martin, N. C., Scourfield, J., \& McGuffin, P. (2009). Genetic overlap between measures of hyperactivity/inattention and mood in children and adolescents. Journal of the American Academy of Child and Adolescent Psychiatry, 48, 1094-1101.

Eaves, L. J. (1988). Dominance alone is not enough. Behavior Genetics, 18, 27-33.

Faraone, S. V., Biederman, J., \& Mick, E. (2006). The age-dependent decline of attention deficit hyperactivity disorder: A meta-analysis of follow- up studies. Psychological Medicine, 36, 159-165.

Faraone, S. V., \& Mick, E. (2011). Molecular genetics of Attention Deficit Hyperactivity Disorder. The Psychiatric Clinics of North America, 33, 159-180.

Freitag, C. M., Rohde, L. A., Lempp, T., \& Romanos, M. (2010). Phenotypic and measurement influences on heritability estimates in childhood ADHD. European Child and Adolescent Psychiatry, 19, 311-323.

Goodman, R. (1997). The strengths and difficulties questionnaire: A research note. Journal of Child Psychology and Psychiatry, 38, 581-586.

Hay, D. A., Bennett, K. S., Levy, F., Sergeant, J., \& Swanson, J. (2007). A twin study of attention-deficit/hyperactivity disorder dimensions rated by the strengths and weaknesses of ADHD-symptoms and normal-behavior (SWAN) scale. Biological Psychiatry, 61, 700-705.

Hicks, B. M., South, S. C., DiRago, A. C., Iacono, W. G., \& McGue, M. (2009). Environmental adversity increases genetic risk for externalizing disorders. Archives of General Psychiatry, 66, 640-648.

Hur, Y-M., Jeong, H-U., Chung, K. W., Shin, J. S., \& Song, T-B. (2013). The South Korean twin registry: An update. Twin Research and Human Genetics, 16, 237-240.

Hur, Y.-M., \& Song, T-B. (2009). A recent rise in twin birth rates in South Korea and demographic characteristics of mothers of twins: 2003-2007. Twin Research and Human Genetics, 10, 373-378.

Kan, K.-J., Dolan, C. V., Nivard, M. G., Middeldorp, C. M., van Beijsterveldt, C. E. M., Willemsen, G., ... Boomsma, D. I. (2013). Genetic and environmental stability in attention problems across the life span: Evidence from the Netherlands Twin Register Journal of the American Academy of Child and Adolescent Psychiatry, 52, 12-25.

Kan, K-J, van Beijsterveldt, C. E. M., Bartels, M., \& Boomsma, D. I. (2014). Assessing Genetic influences on behavior: Informant and context dependency as illustrated by the analysis of attention problems. Behavior Genetics, 44, 326336.

Kerekes, N., Brändström, S., Lundström, S., Råstam, M., Nilsson, T., \& Anckarsäter, H. (2013). ADHD, autism spectrum disorder, temperament, and character: Phenotypical associations and etiology in a Swedish childhood twin study. Comprehensive Psychiatry, 54, 1140-1147.

Kuntsi, J., Eley, T. C., Taylor, A., Hughes, C., Asherson, P., Caspi, A., \& Moffitt, E. E. (2004). Co-occurrence of ADHD and low IQ has genetic origins. American Journal of Medical Genetics. 124B, 41-47.

Kuntsi, J., Rijsdijk, F., Ronald, A., Asherson, P., \& Plomin, R. (2005). Genetic influences on the stability of attention-deficit/hyperactivity disorder symptoms from early to middle childhood. Biological Psychiatry, 57, 647654.

Larsson, J. O., Larsson,, H., \& Lichtenstein, P. (2004). Genetic and environmental contributions to stability and change of ADHD symptoms between 8 and 13 years of age: A longitudinal twin study. Journal of the American Academy of Child and Adolescent Psychiatry, 43, 1267-1275. 
Nadder, T. S., Rutter, M., Silberg, J. L., Maes, H. H., \& Eaves, L. J. (2002). Genetic effects on the variation and covariation of attention deficit-hyperactivity disorder (ADHD) and oppositional-defiant disorder, conduct disorder symptomatologies across informant and occasion of measurement. Psychological Medicine, 32, 39-53.

Neale, M. C., Boker, S. M., Xie, G., \& Maes, H. H. (2003). $M x$ : Statistical modeling (5th ed.,). Richmond, VA: Virginia Commonwealth University, Department of Psychiatry.

Nikolas, M. A., \& Burt, A. S. (2010). Genetic and environmental influences on ADHD symptom dimensions of inattention and hyperactivity: A meta-analysis. Journal of Abnormal Psychology, 119, 1-17.

Ooki, S., Yamada, K., \& Asaka, A. (1993). Zygosity diagnosis of twins by questionnaire for twins' mothers. Acta Geneticae Medicae et Gemellologiae, 42, 17-22.

Plomin, R., DeFries, J. C., McClearn, G. E., \& McGuffin, P. (2001). Behavioral genetics (4th ed.). New York: Worth Publishers and W. H. Freeman and Company.

Polanczyk, G., de Lima, M. S., Horta, B. L., Biederman, J., \& Rohde, L. A. (2007). The worldwide prevalence of ADHD: A systematic review and meta regression analysis. The American Journal of Psychiatry, 164, 942-948.

Purcell, S. (2002). Variance components models for geneenvironment interaction in twin analysis. Twin Research, $5,554-571$.

Rietveld, M. J., Posthuma, 1. D., Dolan, C. V., \& Boomsma, D. I. (2003). ADHD: Sibling interaction or dominance: An evaluation of statistical power. Behavior Genetics, 33, 247255.

Rietveld, M. J. H., Hudziak, J. J., Bartels, M., van Beijsterveldt, C. E. M., \& Boomsma, D. I. (2004). Heritability of attention problems in children: Longitudinal results from a study of twins, age 3 to12. Journal of Child Psychology and Psychiatry, 45, 577-588.

Robbers, S. C. C., van Oort, F. V. A., Polderman, T. J. C., Bartels, M., Boomsma, D. I., Verhulst, F. C., Lubke, G. H., Huizink, A. C. (2011). Trajectories of CBCL attention problems in childhood. European Child and Adolescent Psychiatry, 20, 419-427.

Saudino, K. J., Cherny, S. S., \& Plomin, R. (2000). Parent ratings of temperament in twins: Explaining the 'too low' DZ correlations. Twin Research, 4, 224-233.

Simonoff, E., Pickles, A., Hervas, A., Silberg, J. L., Rutter, M., \& Eaves, L. (1998). Genetic influences on childhood hyperactivity: Contrast effects imply parental rating bias, not sibling interaction. Psychological Medicine, 28, 825-837.

Stone, L. L., Otten, R., Engels, C. M. E., Vermulst, A. A., \& Janssens, M. A. M. (2010). Psychometric properties of the parent and teacher versions of the strengths and difficulties questionnaire for 4- to 12-year-olds: A review. Clinical Child and Family Psychology Review, 13, 254-274.

Taylor, E. (1994). Syndromes of attention deficit and overactivity. In M. Rutter, E Taylor, \& L. Hersov (Eds.), Child and adolescent psychiatry: Modern approaches. Oxford: Blackwell Scientific Publications.

Thapar, A., Cooper, M., Jefferies, R., \& Stergiakouli, E. (2012). What causes attention deficit hyperactivity disorder? Archives of Disease in Childhood, 97, 260-265.

Willcutt, E. G., Pennington, B. F., Chhabildas, N. A., Friedman, M. C., \& Alexander, J. (1999) Psychiatric comorbidity associated with DSM-IV ADHD in a nonreferred sample of twins. Journal of American Academy of Child and Adolescent Psychiatry, 38, 1355-1362. 